\section{Investigation of thermal neutron detection capability of a CdZnTe detector in a mixed gamma-neutron radiation field}

Haluk Yücel, R. Bora Narttürk, Senem Zümrüt, Gizem Gedik, Mustafa Karadag

Abstract. The aim of this study was to investigate the thermal neutron measurement capability of a CdZnTe detector irradiated in a mixed gamma-neutron radiation field. A CdZnTe detector was irradiated in one of the irradiation tubes of $\mathrm{a}^{241} \mathrm{Am}$-Be source unit to determine the sensitivity factors of the detector in terms of peak count rate (counts per second [cps]) per neutron flux (in square centimeters per second) $\left[\mathrm{cps} /\right.$ neutron $\cdot \mathrm{cm}^{-2} \cdot \mathrm{s}^{-1}$ ]. The CdZnTe detector was covered in a 1-mm-thick cadmium (Cd) cylindrical box to completely absorb incoming thermal neutrons via ${ }^{113} \mathrm{Cd}(\mathrm{n}, \gamma)$ capture reactions. To achieve, this $\mathrm{Cd}$-covered $\mathrm{CdZnTe}$ detector was placed in a well-thermalized neutron field $(f$-ratio $=50.9 \pm 1.3)$ in the irradiation tube of the ${ }^{241} \mathrm{Am}-\mathrm{Be}$ neutron source. The gamma-ray spectra were acquired, and the most intense gamma-ray peak at $558 \mathrm{keV}(0.74 \gamma / \mathrm{n})$ was evaluated to estimate the thermal neutron flux. The epithermal component was also estimated from the bare $\mathrm{CdZnTe}$ detector irradiation because the epithermal neutron cutoff energy is about $0.55 \mathrm{eV}$ at the 1-mm-thick $\mathrm{Cd}$ filter. A high-density polyethylene moderating cylinder box can also be fitted into the $\mathrm{Cd}$ filter box to enhance thermal sensitivity because of moderation of the epithermal neutron component. Neutron detection sensitivity was determined from the measured count rates from the $558 \mathrm{keV}$ photopeak, using the measured neutron fluxes at different irradiation positions. The results indicate that the CdZnTe detector can serve as a neutron detector in mixed gamma-neutron radiation fields, such as reactors, neutron generators, linear accelerators, and isotopic neutron sources. New thermal neutron filters, such as Gd and Tb foils, can be tested instead of the Cd filter due to its serious gamma-shielding effect.

Keywords: neutron detection $\bullet \mathrm{CdZnTe} \bullet$ prompt gamma ray $\bullet$ thermal neutron $\bullet$ cadmium $\bullet$ neutron sensitivity ${ }^{241} \mathrm{Am}$-Be source

H. Yücel ${ }^{\bowtie}$, R. B. Narttürk, S. Zümrüt, G. Gedik Institute of Nuclear Sciences

Ankara University

Dogol St., Tandogan, Ankara 06100, Turkey

E-mail: haluk.yucel@ankara.edu.tr

\section{Karadag}

Gazi University

Teknikokullar, Ankara 06100, Turkey

Received: 10 November 2017

Accepted: 29 August 2018

\section{Introduction}

Neutron detection and counting comprise a crucial and multidisciplinary issue in nuclear measurement [1]. Therefore, neutron measurement is still an active subject of research driven by the necessity to find solutions to the ${ }^{3} \mathrm{He}$ shortage in gas detectors and portable neutron dosimeters. The new types of neutron detectors usually take advantage of high-cross-section-capture reactions, inducing charged ions easily separable from recoil electrons. For instance, ${ }^{113} \mathrm{Cd}(\mathrm{n}, \gamma)$ and ${ }^{157} \mathrm{Gd}(\mathrm{n}, \gamma)$ reactions have the highest capture cross-sections for thermal neutrons. However, the prompt gamma rays produced from the radiative capture cannot still be easily discriminated from background gamma rays, especially when the detector is exposed to a mixed gamma-neutron radiation field [2]. In recent years, in particular, robust gamma-ray rejection and promising neutron sensitivity have been obtained in scintillation technology, addressing large-sensor applications [3]. To develop portable detectors addressing personal neutron dosimetry, semiconductor technologies are also 


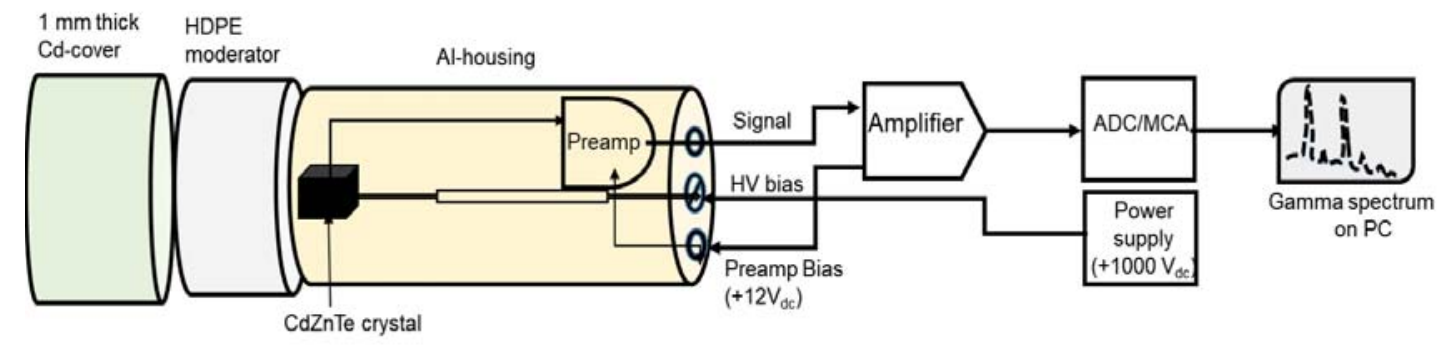

Fig. 1. A schematic measurement assembly of a $5 \times 5 \times 5 \mathrm{~mm}^{3} \mathrm{CdZnTe}$ detector with 1 -mm-thick Cd and high-density polyethylene (HDPE) covers.

preferable due to the high photon-stopping power. Initially, the intrinsic properties of CdTe for neutron detection have been studied [4]. A Gd-covered CdTe diode has been developed and tested using both ${ }^{113} \mathrm{Cd}$ and ${ }^{157} \mathrm{Gd}$ isotopes [5]. Additionally, neutron detection has also been tested using a $25-\mu \mathrm{m}$ -thick Gd foil-covered small CdZnTe sensor (about $0.5 \mathrm{~cm}^{3}$ ) in the low-energy range of $0-200 \mathrm{keV}$ using the compensation mode [6]. To make a portable neutron detector, a $0.5 \mathrm{~cm}^{3} \mathrm{CdZnTe}$ diode has been successively used without converter, with $\mathrm{Gd}$ converter and $\mathrm{Tb}$ converter foils having thicknesses equal to $5 \mu \mathrm{m}$. In this case, a Tb foil was chosen to increase the signal in the range of $60-200 \mathrm{keV}$ because the atomic number $Z=65$ for $\mathrm{Tb}$ is close to $Z=64$ for $\mathrm{Gd}$ [2].

Among the wide-bandgap detectors, CdZnTe detectors hold promise as practical and efficient radiation spectroscopy devices. In particular, $\mathrm{CdZnTe}$ detectors are of great interest for room temperature X-ray and gamma-ray spectroscopy. Hence, in recent years, CdZnTe detectors have been commonly used in medicine, environmental remediation, and physics research [7-9]. The spectroscopic performances of large-volume coplanar grid $\mathrm{CdZnTe}$ detectors have great potential in gamma-ray spectroscopy due to their mainly improved energy resolution [10]. In parallel with technological developments, larger-volume crystals have been grown, thus providing higher detection efficiency of gamma rays and neutrons. The $\mathrm{CdZnTe}$ crystal has already been evaluated regarding whether it can be used as a neutron detector in medical accelerators. The neutron counting efficiency of the CdZnTe detector has been estimated using the prompt $558 \mathrm{keV}$ photopeak, following the ${ }^{113} \mathrm{Cd}(\mathrm{n}, \gamma)$ capture reaction. It showed good neutron detection performance at about $10^{4}$ neutron $\cdot \mathrm{cm}^{-2} \cdot \mathrm{s}^{-1}$ fluxes in medical accelerators [11].

The detection principle is based on the ${ }^{113} \mathrm{Cd}(\mathrm{n}, \gamma){ }^{114} \mathrm{Cd}$ reaction. It emits several prompt gamma rays within time periods of $10^{-12}-10^{-14} \mathrm{~s}$. The most intense gamma ray is of $558 \mathrm{keV}(0.74 \mathrm{\gamma} / \mathrm{n})$ and the second-intense gamma ray is of $651 \mathrm{keV}$ $(0.14 \gamma / \mathrm{n})$. The recommended thermal cross-section of ${ }^{113} \mathrm{Cd}$ (its abundance is $12.4 \%$ ) is very high, i.e., $20615 \pm 400$ barns [12]. In this study, thermal neutrons produced from a water-moderated $37 \mathrm{GBq}$ ${ }^{241} \mathrm{Am}$-Be neutron source is used for the irradiation. For the characterization of the neutron field in the irradiation unit, first, thermal neutron fluxes were measured at different irradiation positions by the foil activation method using ${ }^{197} \mathrm{Au}(\mathrm{n}, \gamma){ }^{198} \mathrm{Au}$ monitor reaction. The neutron-induced gamma-ray spectra of the irradiated Au monitor were measured in the well of a p-type high-purity germanium (HPGe) detector, and then they were evaluated to determine neutron flux. Then, a CdZnTe detector was positioned at the same position at the known neutron flux.

The purpose of the present study is to investigate CdZnTe semiconductor detectors that can be used for dual purposes. The motivation for this study is to analyse whether these detectors are suitable for the measurement of both neutron dose rate as well as gamma dose rate at the same time.

\section{Materials and methods}

In this study, as seen in Fig. 1 , a $5 \times 5 \times 5 \mathrm{~mm}^{3}$ CdZnTe-based, room temperature, single point extended area radiation (SPEAR) detector (Spear Products Inc., PA, USA), either containing a 1-mm-thick Cd filter box or without the Cd filter (bare detector), is located at the appropriate position in the neutron source irradiation facility to study the exposure to the gamma and neutron fields for given periods. The CdZnTe detector was irradiated in the central irradiation tube of the ${ }^{241} \mathrm{Am}$-Be source unit to determine the neutron sensitivity of the CdZnTe detector in terms of peak count rate (counts per second [cps]) per neutron flux (in square centimeters per second) [cps/neutron $\left.\cdot \mathrm{cm}^{-2} \cdot \mathrm{s}^{-1}\right]$. The CdZnTe detector was first fitted into a 1-mm-thick $\mathrm{Cd}$ cylindrical box, to completely absorb the incoming thermal neutrons, to measure the epithermal neutron component only, because the epithermal neutron cutoff energy is $\sim 0.55 \mathrm{eV}$ in a 1 -mm-thick Cd filter $[14,15]$. The irradiations were performed at the different chosen positions in a Cd-covered CdZnTe detector; a well-thermalized neutron field exists in the central irradiation tube of the ${ }^{241} \mathrm{Am}-\mathrm{Be}$ neutron source. The gamma-ray spectra were acquired and then the area of the $558 \mathrm{keV}(0.74 \gamma / \mathrm{n})$ peak, which is the most intense prompt gamma ray, was evaluated to estimate the thermal neutron flux. The thermal plus epithermal components were estimated from the bare CdZnTe detector (without Cd cover) irradiation. A high-density polyethylene (HDPE; 5-mm thickness) cover can be used for providing extra thermalization of the epithermal component in the case of Cd-covered detector irradiations. The measured count rates were determined from the prominent 


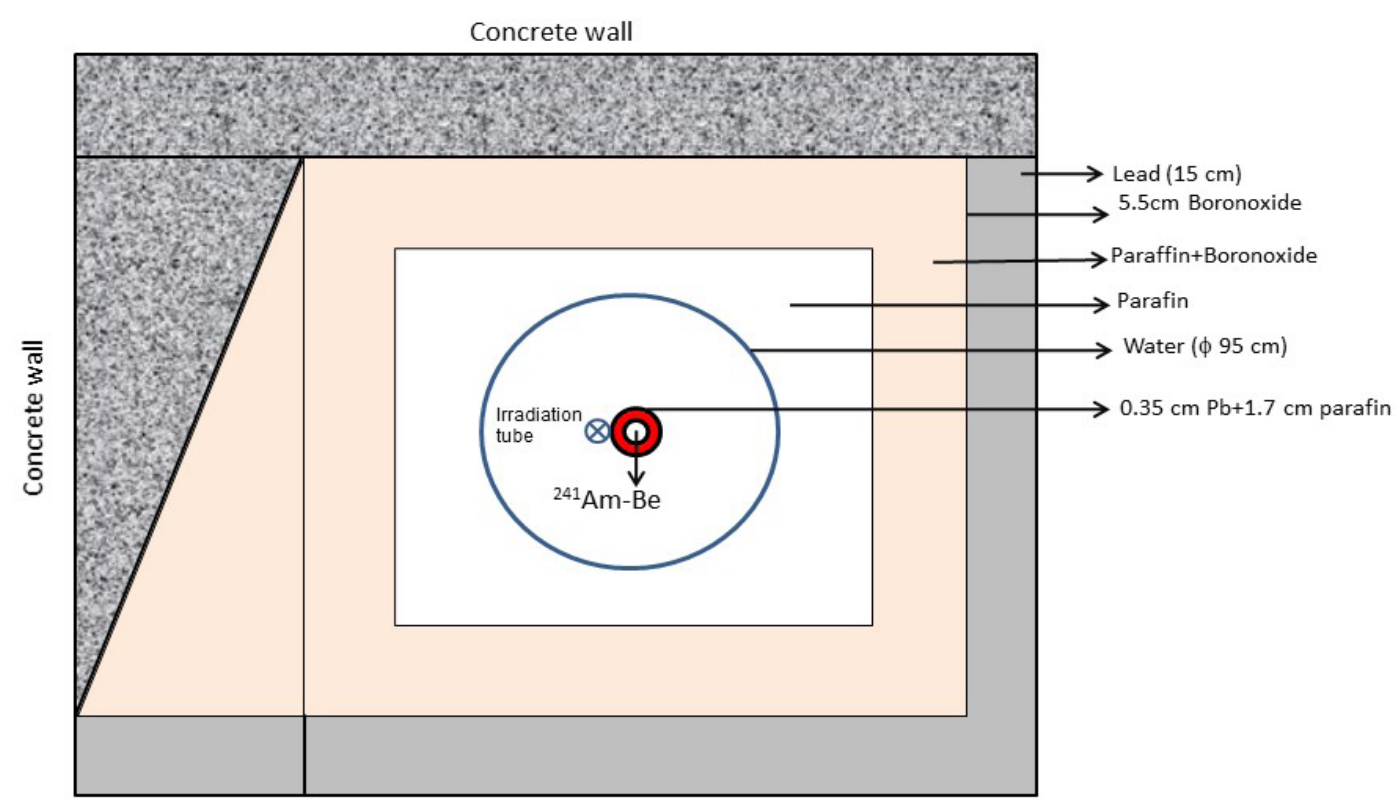

Fig. 2. A schematic diagram of the neutron irradiation unit with a $37 \mathrm{GBq}^{241} \mathrm{Am}-\mathrm{Be}$ source [13].

$558 \mathrm{keV}$ and $651 \mathrm{keV}$ photopeaks of the ${ }^{114} \mathrm{Cd}$ prompt gamma rays, using the measured neutron fluxes at the relevant irradiation positions.

It is essential that a reliable neutron flux measurement method is chosen for the validation of flux measurement at the interested irradiation position. To do this, thermal neutrons produced from a water-moderated $37 \mathrm{GBq}^{241} \mathrm{Am}$-Be neutron source (Fig. 2) were measured by the foil activation method using a dual monitor via ${ }^{197} \mathrm{Au}(\mathrm{n}, \gamma){ }^{198} \mathrm{Au}$ and ${ }^{55} \mathrm{Mn}(\mathrm{n}, \gamma){ }^{56} \mathrm{Mn}$ reactions. The used foils have high purity of $99.95 \%$, with $0.0508 \mathrm{~mm}(0.002 \mathrm{in})$ thickness and small diameters of $12.7 \mathrm{~mm}$ (0.5 in) (purchased from Shieldwerx, a division of Bladewerx LLC, Rio Rancho, NM, USA), to minimize the thermal neutron self-shielding factor, $G_{t h}$, as well as the epithermal neutron self-shielding factor $G_{e p i}$. The characterization of the ${ }^{241} \mathrm{Am}-\mathrm{Be}$ neutron irradiation unit is already described in detail [13]. The neutron-induced gamma-ray spectra were measured in the well of a p-type, $44.8 \%$ relative-efficiency HPGe detector (Model GCW4023; Canberra Inc.). The Ge detector has a well with $16-\mathrm{mm}$ diameter and $40-\mathrm{mm}$ depth. It has a standard $10-\mathrm{cm}$-thick $\mathrm{Pb}$ shield, graded by 1-mm-thick $\mathrm{Sn}$ and 1.6-mm-thick Cu layers, jacketed by a $9.5-\mathrm{mm}$ steel outer housing. The detector was modelled by Monte Carlo simulation (GESPECOR 3.2) and ANGLE 4 software efficiency transfer (ET) method to determine the efficiency calibration curve for a well geometry. In the ET method, reference efficiency curves were determined experimentally from standard sources, namely, ${ }^{241} \mathrm{Am},{ }^{57} \mathrm{Co},{ }^{54} \mathrm{Mn}$, ${ }^{60} \mathrm{Co},{ }^{65} \mathrm{Zn},{ }^{109} \mathrm{Cd},{ }^{133} \mathrm{Ba},{ }^{137} \mathrm{Cs}$, and ${ }^{22} \mathrm{Na}$ (purchased from Eckert and Ziegler Inc.).

\section{Results and discussion}

Eight different irradiation positions were determined from the reference position marked at the top of the central neutron irradiation tube, and then the gamma- -ray spectra were acquired by a $5 \times 5 \times 5 \mathrm{~mm}^{3}$ $\mathrm{CdZnTe}$ detector placed at these heights. The prompt gamma rays, e.g., 558 and $651 \mathrm{keV}$, were observed clearly as analytical peaks above the source background, as shown in Fig. 3a. The continuum background counts are due to mostly elastic and inelastic scattering neutrons, and the ones originating from the neutron source itself as well as the used shielding and moderating materials, e.g., ${ }^{1} \mathrm{H}(\mathrm{n}, \gamma)$ capture reaction in hydrogenous materials such as water and paraffin, which are heavily used in the irradiation unit. The observed 558 and $651 \mathrm{keV}$ peaks from the reaction ${ }^{113} \mathrm{Cd}(\mathrm{n}, \gamma){ }^{114} \mathrm{Cd}$, caused by the interaction of the thermal and epithermal neutrons passing through the CdZnTe detector, are easily quantified to get the net counts. The measurement results for the count rates for the $558 \mathrm{keV}$ peak are given in Table 1 . The neutron fluxes at different irradiation points measured with gold foils through ${ }^{197} \mathrm{Au}(\mathrm{n}, \gamma){ }^{198} \mathrm{Au}$ monitor reaction are also given in Table 1 . The thermal-to-epithermal flux ratio $(f)$ of $50.9 \pm 1.3$, as given in the study by Yücel et al. [13], was used to estimate the epithermal neutron sensitivity. The peak count rates were obtained both with and without the $\mathrm{Cd}$ box. The last column of Table 1 shows the percentage thermal-to-epithermal neutron sensitivity of the CdZnTe detector. This means that 1-mm-thick Cd filter totally absorbs the thermal neutrons but the epithermal neutrons interact with the CdZnTe crystal. Therefore, the thermal neutron sensitivity is always lower than the epithermal neutron sensitivity of the detector, that is, on the average, $4.8 \%$. This implies that the Cd-filter box serves as a shield against the gamma rays, such as those of energy 558 and $651 \mathrm{keV}$, produced in it. In the data analysis, we have focused on the most intense gamma-ray peak region $(558 \mathrm{keV})$ to accurately determine the difference in peak count rate between the results with and without the Cd box. An example of the obtained spectra is shown in Fig. 3b. This difference is remarkable because of the complete absorption of the thermal 
a

b

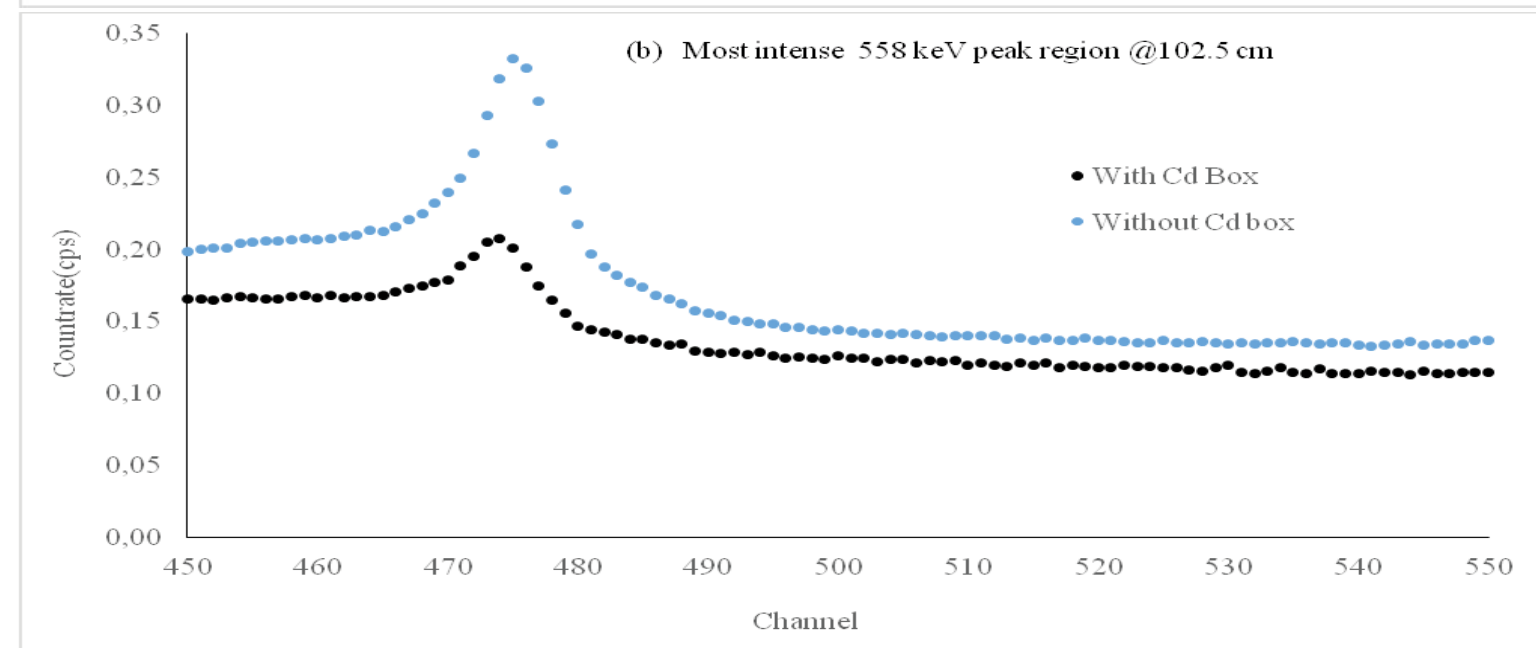

(a) Full spectra of CdZnTe detector $@ 102.5 \mathrm{~cm}$

- Without Cd box

- With Cd box

Fig. 3. Prompt gamma-ray spectra of $\mathrm{CdZnTe}$ detector with and without $\mathrm{Cd}$ taken in the Am-Be neutron irradiation unit: (a) full spectra and (b) most intense peak $558 \mathrm{keV}$.

neutrons in the 1-mm-thick Cd box. In this configuration, it is clear that the fraction of the counted gamma rays is related to the solid angle. That is, we have to be consider the number of neutrons crossing a surface of area $S$ (a cube-shaped detector: $\left.6 \times 0.5 \times 0.5=0.75 \mathrm{~cm}^{2}\right)$, and this can be estimated to be $[(S / 4) \times$ neutron flux density $\times$ absolute detection efficiency $\times 74$ prompt gamma/100] for the $558 \mathrm{keV}$ peak if we correctly determine the absolute efficiency. However, for instance, some of the $558 \mathrm{keV}$ gamma rays may escape from the detector or losses may occur due to the true coincidence when summing with other gamma rays. Therefore, experimental measurement of neutron detection needs to be conducted for the CdZnTe detector. Nevertheless, in future work, a Monte Carlo simulation of the irradiation configuration and the measurement setup using the MCC-MT software (for 3D modelling of the processes of transfer and registration of ionizing radiation) will be made to investigate the effect of filters made of different materials, such as Gd and $\mathrm{Tb}$, besides $\mathrm{Cd}$.

\section{Conclusion}

This study indicates that a CdZnTe detector can measure both thermal neutrons and gamma rays in the mixed field. The neutron detection capability of a CdZnTe detector can be defined as the neutron measurement sensitivity in units of count rates per neutron flux [cps/neutron $\cdot \mathrm{cm}^{-2} \cdot \mathrm{s}^{-1}$, where the $558 \mathrm{keV}$ peak count rate (cps) is measured. The Cd cover was intentionally chosen as the neutron converter through ${ }^{113} \mathrm{Cd}(\mathrm{n}, \gamma)$ reactions. However, the results given in Table 1 imply that the 1-mm-thick Cd cover itself serves as a good gamma shield against the gamma rays, such as those of energy $558 \mathrm{keV}$, produced in it. Hence, it is worth noting that the Cd filter remarkably reduced the $558 \mathrm{keV}$ 


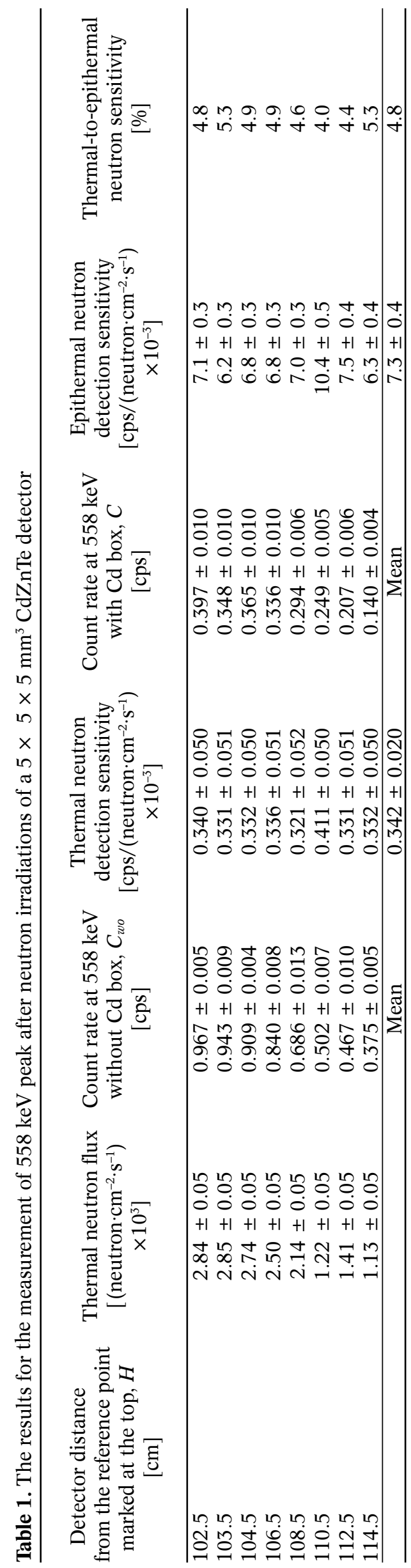

peak count rates arising from thermal neutrons in the mixed field. Thus, this resulted in the lowering of the thermal neutron detection sensitivity of the $\mathrm{CdZnTe}$ detector used herein. To examine this effect on the thermal neutron sensitivity, thermal neutrons incoming on the CdZnTe detector might be absorbed by another filter such as Gd or Tb. However, in this study, when we used a 1-mm-thick Cd cylindrical cover around the CdZnTe detector, the thermal neutron-to-epithermal neutron sensitivity was estimated to be $4.8 \%$. In other words, epithermal neutron detection is better than thermal neutron detection. Epithermal neutron detection can be further enhanced by the use of a 5-mm HDPE moderator fitted into a 1-mm-thick Cd filter, for ensuring extra thermalization of the epithermal and fast neutrons coming from the source. In fact, it is an interesting point that thermal neutron sensitivity loss is expected due to the thermal neutrons not reaching the detector crystal and also because some of the prompt gamma rays from the neutron capture reactions in the Cd-shield box cannot reach the detector crystal. Hence, a small peak of $558 \mathrm{keV}$ normally appears in the whole gamma spectrum, as shown in Fig. 3. This suggests that the Cd cover itself remarkably shields the gamma photons produced in the unit. The present results show that a CdZnTe semiconductor can be used as a versatile neutron detection device in mixed gamma-neutron radiation fields. The proper assembly of a CdZnTe detector can serve as an active dosimeter to read the received doses due to both neutrons and gamma rays.

In future work, in order to test the thermal and epithermal neutron detection sensitivity of a $\mathrm{CdZnTe}$ detector through ${ }^{155} \mathrm{Gd}(\mathrm{n}, \gamma)$ and ${ }^{157} \mathrm{Gd}(\mathrm{n}, \gamma)$, Gd and $\mathrm{Tb}$ foils will be used for wrapping a coplanar grid of $10 \times$ $10 \times 10 \mathrm{~mm}^{3} \mathrm{CdZnTe}$. Gamma suppression effect of the Gd or Tb cover box on the $558 \mathrm{keV}$ analytical peak will be compared with that of the $\mathrm{Cd}$ box. Additional $\mathrm{Pb}$ and $\mathrm{W}$ foils will have beneficial effect as gamma attenuator foils surrounding the $\mathrm{CdZnTe}$ detector.

For future works, a calibration study will be made for calibration of dose rate conversion of the whole spectrum and the measured count rates for high-intensity $558 \mathrm{keV}$ peak from the ${ }^{241} \mathrm{Am}-\mathrm{Be}$ neutron source. This may provide information about whether a CdZnTe detector can be used as a neutron dose rate meter or not. The present CdZnTe detector will be exposed to the mixed field under the head of a $18 \mathrm{MV}$ linac in the radiotherapy room to test its neutron detection capability to demonstrate the potential use of a $\mathrm{CdZnTe}$ detector as an active neutron dose meter.

It seems that $\mathrm{CdZnTe}$ has promising potential for use in the operation of reactors, neutron generators, linacs, and cyclotrons or isotopic neutron sources, such as ${ }^{241} \mathrm{Am}-\mathrm{Be},{ }^{238} \mathrm{Pu}-\mathrm{Be}$, etc., which can produce a mixed gamma-neutron radiation field for various purposes.

Acknowledgments. This study was supported by the TÜBİTAK project grant number $116 \mathrm{~F} 308$ and partly by the Ankara University BAP Project 12A4045001. 
It was submitted as a poster presentation titled "Investigation of thermal neutron detection capability of a CdZnTe detector in a mixed gamma-neutron field" in the International Conference on Developments and Applications of Nuclear Technologies (NUTECH 2017), September 10-13, 2017, held in Krakow, Poland.

\section{References}

1. Dumazert, J., Coulon, R., Lecomte, Q., Bertrand, G. H. V., \& Hamel, M. (2018). Gadolinium for neutron detection in current nuclear instrumentation research: A review. Nucl. Instrum. Methods Phys. Res. Sect. A-Accel. Spectrom. Dect. Assoc. Equip., 882, 53-68.

2. Coulon, R., Dumazert, J., Hamel, M., Bertrand, G., Carrel, F., Kondrasovs, V., \& Boudergui, K. (2016). Implementation of gadolinium for neutron measurement systems based on plastic scintillators or semiconductors. In IEEE NSS Symposium Proceedings, 2016 IEEE Nuclear Science Symposium, Medical Imaging Conference and Room-Temperature Semiconductor Detector Workshop (NSS/MIC/RTSD) (pp. 1-6). Strasbourg.

3. Dumazert, J., Coulon, R., Bertrand, G. H. V., Normand, S., Mechin, L., \& Hamel, M. (2016). Compensated bismuth-loaded plastic scintillators for neutron detection using low-energy pseudospectroscopy. Nucl. Instrum. Methods Phys. Res. Sect. A-Accel. Spectrom. Dect. Assoc. Equip., 819, 25-32.

4. Fasasi, M., Jung, M., Siffert, P., \& Teissier, C. (1988). Thermal neutron dosimetry with cadmium telluride detectors. Radiat. Prot. Dosim., 23, 429-431.

5. Miyake, A., Nishioka, T., Singh, S., Morii, H., Mimura, H., \& Aoki, T. (2011). A CdTe detector with a Gd converter for thermal neutron detection. Nucl. Instrum. Methods Phys. Res. Sect. A-Accel. Spectrom. Dect. Assoc. Equip., 654, 390-393.

6. Dumazert, J., Coulon, R., Kondrasovs, V., \& Boudergui, K. (2017). Compensation scheme for online neutron detection using a Gd-covered $\mathrm{CdZnTe}$ sensor. Nucl. Instrum. Methods Phys. Res. Sect. A-Accel. Spectrom. Dect. Assoc. Equip., 857, 7-15.
7. Schlesinger, T. E., \& James, R. B. (1995). Semiconductors for room temperature nuclear detector applications (Vol. 43). Series Semiconductors and Semimetals. New York: Academic Press.

8. He, Z., Knoll, G. K., Wehe, D. K., \& Miyamoto, J. (1997). Position sensitive single carrier CdZnTe detectors. Nucl. Instrum. Methods Phys. Res. Sect. A-Accel. Spectrom. Dect. Assoc. Equip., 388, 180-185.

9. Yücel, H., Uyar, E., \& Esen, A. N. (2012). Measurements on the spectroscopic performance of CdZnTe coplanar grid detectors. Appl. Radiat. Isot., 70, 1608-1615. DOI: 10.1016/j.apradiso.2012.04.027.

10. González, R., Pérez, J. M., Vela, O., de Burgos, E., Oller, J. C., \& Gostilo, V. (2005). Spectrometric response of large volume CdZnTe coplanar detectors. IEEE Trans. Nucl. Sci., 52(5), 2076-2084. DOI: 10.1109/TNS.2005.856887.

11. Martín, A. M., Iñiguez, M. P., Luke, P. N., Barquero, R., Lorente, A., Morchón, J., Gallego, E., Quincoces, G., \& Martí-Climent, J. M. (2009). Evaluation of CdZnTe as neutron detector around medical accelerators. $R a$ diat. Prot. Dosim., 133(4), 193-199. DOI: 10.1093/ $\mathrm{rpd} / \mathrm{ncp} 038$.

12. EXFOR Database. (2017). https://wwwnds.iaea. org/exfor/servlet/X4sGetSubent? reqx=548\&sub ID=311001490 (Access date: 7 September 2017).

13. Yücel, H., Budak, M. G., Karadag, M., \& Yuksel, A. O. (2014). Characterization of neutron flux spectra in the irradiation sites of a $37 \mathrm{GBq}^{241} \mathrm{Am}-\mathrm{Be}$ isotopic source. Nucl. Instrum. Methods Phys. Res. Sect. B-Beam Interact. Mater. Atoms, 338, 139-144. DOI: 10.1016/j. nimb.2014.08.010.

14. Yücel, H., \& Karadag, M. (2004). Experimental determination of the $\alpha$-shape factor in the $1 / \mathrm{E}^{1+\alpha}$ epithermal-isotopic neutron source-spectrum by dual monitor method. Ann. Nucl. Energy, 31(6), 681-695.

15. Karadag, M., Yücel, H., Tan, M., \& Özmen, A. (2003). Measurement of thermal neutron cross-sections and resonance integrals for ${ }^{71} \mathrm{Ga}(\mathrm{n}, \gamma){ }^{72} \mathrm{Ga}$ and ${ }^{75} \mathrm{As}(\mathrm{n}, \gamma){ }^{76} \mathrm{As}$ by using ${ }^{241} \mathrm{Am}-\mathrm{Be}$ isotopic neutron source. Nucl. Instrum. Methods Phys. Res. Sect. A-Accel. Spectrom. Dect. Assoc. Equip., 501(2/3), 524-535. 